\title{
GENERATION OF HIGH-RESOLUTION DIGITAL SURFACE MODELS FOR URBAN FLOOD MODELLING USING UAV IMAGERY
}

\author{
EMRAH YALCIN \\ Department of Civil Engineering, Ahi Evran University, Turkey
}

\begin{abstract}
This study focuses on the generation of a high resolution digital surface model (DSM) using unmanned aerial vehicle (UAV)-based aerial photography to be utilized in the two-dimensional (2D) hydrodynamic model analyses to investigate the effects of possible flood events in the city centre of Kirsehir, Turkey. One of the most important sources of uncertainty in hydrodynamic models is the resolution of used DSM. Changing DSM resolution can cause serious differences in the simulation results about flood extents, water depths, and flow velocities. Therefore, in complex urban areas, high resolution topographic data is a prerequisite to more accurately simulate the movement of water and thereby to take adequate measures for reducing the most likely flood effects. The use of UAV technologies in the production of topographic data is an appropriate solution for situations when airborne light detection and ranging (LiDAR) or satellite-based remote sensing techniques cannot be implemented due to cost, schedule, and resolution issues. Moreover, terrestrial solutions are much more time-consuming and difficult to apply than UAV-based aerial photography in a complex urban area with intensive vehicle and pedestrian traffic. In this study, the topographic data is obtained by using 2212 geo-referenced images taken on 25 different flight routes over an area of $3.24 \mathrm{~km}^{2}$ using a DJI Matrice 600 UAV-drone equipped with a Zenmuse X5 camera with $15 \mathrm{~mm}$ DJI lens. The ground sampling distance of the produced digital surface model is $4.32 \mathrm{~cm} / \mathrm{pixel}$, and the localization accuracy, root mean square error (RMSE), of the model is $5.1 \mathrm{~cm}$ and $12.7 \mathrm{~cm}$ for the horizontal and vertical planes, respectively. The results show that high-resolution digital surface models, which can be used as inputs in 2D flood models for preparing flood hazard and flood risk maps, can be produced with relatively low cost and within a short time using UAV imagery.
\end{abstract}

Keywords: digital surface model, UAV imagery, urban flood modelling, Kirsehir, Turkey.

\section{INTRODUCTION}

Urban floods cause severe destruction of life and property in various parts of the world. To predict and manage such disasters, it is crucial to represent the flow structure of inundation process on a street-by-street basis. Two-dimensional (2D) hydrodynamic models are widely utilized in simulating the inundation dynamics and extent of flood events affecting areas with complex geometry due to their high order of accuracy [1]. One of the most important source of uncertainty influencing the resulting distribution of velocity and water depth over the extent of flooded area built by these simulation models is the quality of inputted terrain data.

Several studies have demonstrated that a high resolution digital surface model (DSM) is needed to accurately simulate the effects of a possible flood event by resolving the detailed and complex flow structure on an urban topography [2]-[6]. Changing DSM resolution can cause serious differences in the simulation results about flood extents, water depths, and flow velocities. Moreover, the vertical accuracy of used DSM is also highly critical to prevent errors in the flooded area calculations [7]. Hence, in complex urban areas, high resolution topographic data with sufficient vertical accuracy is a prerequisite to more accurately simulate the movement of water and thereby to take adequate measures for reducing the most likely flood effects. 
The advances in remote sensing technology make possible to generate high resolution DSMs. High-quality DSMs with vertical accuracies of $10 \mathrm{~cm}$ can be developed using $0.5 \mathrm{~m}$ resolution satellite imagery (e.g., WorldView-2 and GeoEye) [8]. The technology of airborne light detection and ranging (LiDAR) allows to produce $0.1 \mathrm{~m}$ resolution DSMs with vertical accuracies of $25 \mathrm{~cm}$, depending on terrain features, flight parameters, and environmental conditions [1], [8]. Although such data will significantly improve the accuracy of hydraulic models, these technologies are still very expensive to apply in relatively small areas. Another disadvantage of satellite solutions is that the needed imagery cannot be acquired immediately when requested due to their dependence of cloud conditions and revisit time frequencies.

The use of unmanned aerial vehicle (UAV) technology in the production of high quality topographic data is an appropriate solution for areas where manned aircrafts are either not available or would be prohibitively expensive, and satellite solutions do not meet project constraints in terms of cost and schedule. UAV photogrammetry can also be preferable than time-consuming and inapplicable terrestrial solutions in high-risk areas without endangering human operators or in complex urban areas with intensive vehicle and pedestrian traffic. UAV photogrammetric technology allows the acquisition of high-spatial-resolution images from which it is possible to generate a DSM including the objects above bare ground surface as buildings and vegetation at centimetre-scale accuracy. It has been widely utilized in several studies on topographic measurements and monitoring of natural hazards due to its advantages on rapid acquisition of high-spatial-resolution images, cost-effectiveness, and high temporal resolution [9]-[14].

This study focuses on the generation of a high-resolution DSM using UAV-based aerial photography to be utilized in 2D hydrodynamic model analyses to investigate the effects of possible flood events in the city centre of Kirsehir, Turkey. Prior to flight operations, the locations of ground control points (GCPs) are designated in the targeted mapping area. An autonomous flight plan is designed according to the designated departure locations, and the acquired aerial images are processed to generate a high-resolution DSM and an orthophoto of this flood risk zone. The absolute accuracy of the generated DSM is evaluated by comparison with the coordinates of check points selected from the pre-installed GCPs.

\section{STUDY AREA}

Kirsehir province is in the Central Anatolia Region of Turkey. The city centre of Kirsehir faces flood risk from Hastane Creek which comes from the mountainous area in the north of the city. The connection of this intermittent stream to Kilicozu Creek was interrupted by the construction of the Ankara-Kayseri road, and after this point, the stream bed was completely disappeared with intensive construction of buildings and roads in the city centre. Therefore, in a possible flood event, water coming from the mountainous area will pass the intercity road and head directly towards the city centre. The last major flood from this mountainous area occurred in 1989, resulting in the inundation of roads and ground-floors of several buildings in a part of the city centre. However, there is no flood report demonstrating the flood extent related to this event.

It is estimated that the area that could be exposed to a possible flood event from Hastane Creek covers an area of $3.24 \mathrm{~km}^{2}$, which is about $60 \%$ of the city centre (Fig. 1). This flood risk zone is a complex urban region in which there are many critical structures such as hospitals, schools, playgrounds, public institutions, and mosques with intensive vehicle and pedestrian traffic. In this assessment, to investigate the effects of possible flood events in the city centre of Kirsehir, a high resolution digital surface model that can be used as input in flood modelling studies for preparing flood hazard and flood risk maps is generated using UAV-based aerial photography. 


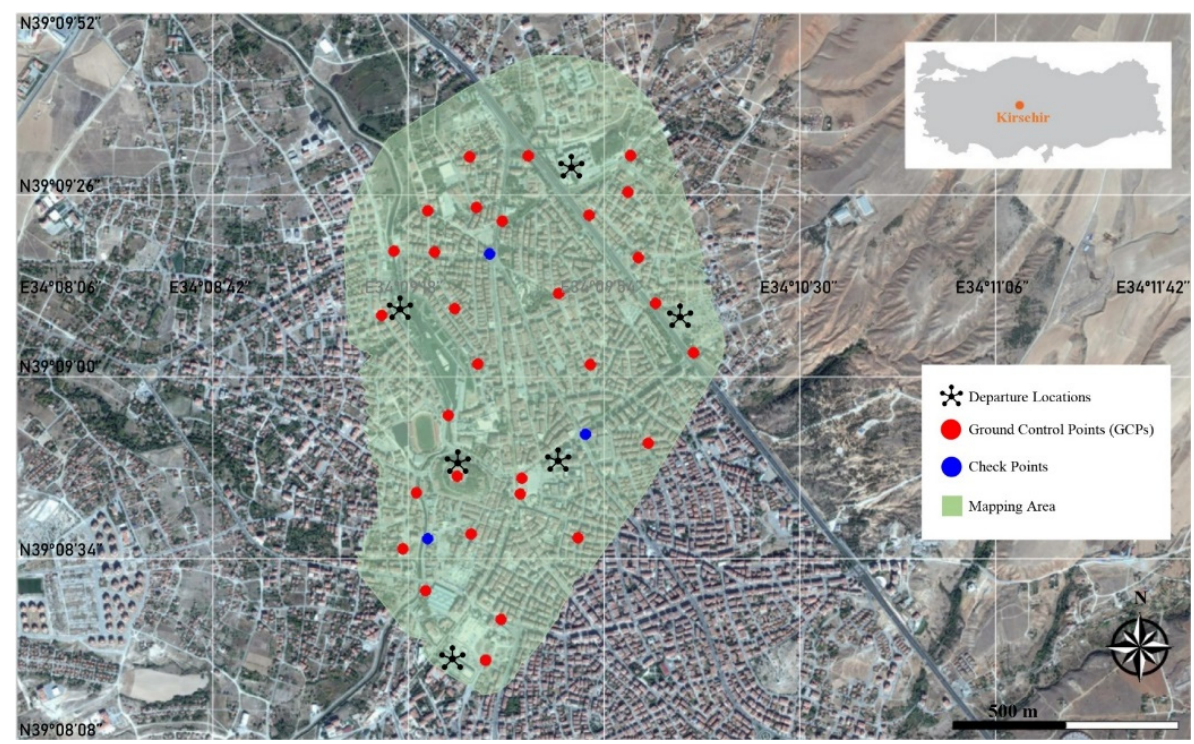

Figure 1: Mapping area and distributions of GCPs, check points, and departure locations.

\section{METHODS}

The framework for the generation of a high-resolution DSM using UAV-based aerial photography consists of three main stages: establishment of GCPs and coordinate measurements, acquisition of aerial images using a rotary-wing UAV, and processing of the acquired aerial images.

\subsection{GCP establishment and coordinate measurement}

Prior to UAV flights for acquiring aerial images, 33 GCPs in the target mapping area are collected that are marked using red spray paint to be identified in the aerial images (Fig. 1). The latitude, longitude and elevation of each point under WGS84/UTM zone 36N coordinate system are measured with a Leica Viva GS15 GNSS receiver [15] using the network of continuously operating reference stations, Turkey (CORS-TR) [16]. 30 of these GCPs are utilized in the process of refining the geolocation of the aerial images, whereas the remaining 3 of them are utilized as check points in evaluating the accuracy of georeferencing process.

\subsection{Aerial photography using a rotary-wing UAV}

Flights are conducted with a DJI Matrice 600 UAV-drone [17] equipped with a Zenmuse X5 camera with $15 \mathrm{~mm}$ DJI lens. This rotary-wing UAV flies using the rotation force of its 6 rotor blades. This design appears to be advantageous against fixed-wing type UAVs in terms of vertical take-off and landing in confined spaces, hovering capability, and capturing vertical and tilted images by changing its orientation [18]. However, rotary-wing UAVs have shorter flight duration than fixed-wing type UAVs because of the need for continuous power during flight operation [19]. Similar to other rotary-wing UAVs, the used model offers about 20-25 min of flight duration on a single battery charge [18].

The used UAV has an autopilot package that allows autonomous flights for predetermined flight paths [17]. Prior to flights, an appropriate flight plan across the target area 
is formed using UgCS software [20] according to the remote-control range of the device and the designated departure locations in the study area (Fig. 1). Moreover, flight characteristics like image overlap percentages, flight altitude, cruise speed and target ground sampling distance are integrated into the flight plan for autonomous navigation and image capture. The UAV is controlled in automatic mode according to the uploaded flight plan with a remote control connected to a smartphone running UgCS mobile application [21] via wireless connection.

The global positioning system (GPS) receiver of the UAV determines its position and altitude by finding GPS satellites, and a compass gathers geomagnetic data, complementing momentary location information [18]. However, image orientation based on only geotags from navigation-grade on-board GPS may lead to 10-20 meters global shift of the coordinates due to atmospheric conditions that cannot be reduced or detected by averaging the geocoded images in image processing [22]. Therefore, the use of GCPs in image georeferencing is strongly recommended to improve the absolute accuracy of the model to be generated [23]. GCPs reduce the shift due to GPS geotags from meters to centimetres, placing the model at the exact position on the Earth [24].

The flight operations are completed in approximately 3 days using 3 sets of battery packs. A total of 2219 geocoded images with $60 \%$ forward and $40 \%$ side overlaps are obtained over the target mapping area from flights conducted on 25 routes at about $120 \mathrm{~m}$ altitude and a speed of $5 \mathrm{~m} / \mathrm{s}$ with departures from 6 different locations (Fig. 1). 3 images out of 2219 (e.g., images taken during take-off and landing, blurred images, etc.) are detected as unusable and discarded from the dataset for image processing. Flight operations are not repeated for the shooting points of these unusable images due to the high overlapping between neighbouring images.

\subsection{Image processing}

Data processing for the acquired 2216 aerial images are conducted using Pix4DMapper Pro software [25] in four steps: (1) initial processing, (2) adding GCPs and validation (3) point cloud and mesh generation, and (4) DSM and orthophoto generation. Before moving into the stages of model generation, the acquired images, image properties about geolocation, camera model associated to the images, output coordinate system, and desired model template are provided as inputs to the software.

As the first step of data processing, an initial processing run is carried out for automatically extracting scale-invariant key points on the images using the structure from motion (SfM) algorithm [26], and automatically connecting these points to each other using cross-comparison analysis of overlapping images [18]. In the second step, the position data of the GCPs are uploaded to the software. In the rayCloud editor of the software, the exact locations of the GCPs are identified on multiple images with the help of the markings made on the side with red spray paint. 3 of these GPSs are defined as check point to test the accuracy of georeferencing process. Then, the camera parameters are reoptimized by bundle block adjustment algorithm to be geometrically corrected the connected key points using the automatic aerial triangulation surveying (AAT) method [27]. As the result of the first two steps of image processing, a sparse point cloud consisting only of points that are successfully matched and verified along multiple images is generated [28]. In the third step, to obtain a much denser point cloud, a point cloud densification process is performed under the following setting options: (1) original image size for image scale, (2) optimal point density, and (3) minimum 3 matches. Then, the generated 3D point cloud with millions of points is triangulated to create a $3 \mathrm{D}$ textured mesh in medium resolution. In the last step of image 
processing, a DSM is generated using the inverse distance weighting method with noise filtering and sharp surface smoothing as DSM filters. Furthermore, an orthophoto of the mapping area is generated by integrating multiple geometrically corrected aerial images using the DSM processing tool of Pix4DMapper Pro software.

\section{RESULTS AND DISCUSSION}

In this study, the topographic data is obtained by using 2216 geo-referenced images taken on 25 different flight routes over an area of $3.24 \mathrm{~km}^{2}$. The number of overlapping images calculated for each pixel across the mapping area is demonstrated in Fig. 2(a). Red, orange, and yellow areas at the boundaries of the target mapping area indicate the overlaps of less than 4 images for each pixel for which poor results may be generated. Green areas indicate an overlap of more than 4 images for every pixel. The results demonstrate that high-quality results could be generated if the number of key points is also appropriate for these green areas. The determined image positions with links between matched images are illustrated in Fig. 2(b). The darkness of the links in the match network indicates the strength of these links. The mean numbers of key points and matched key points per aerial image are 26008 and 5816 , respectively (Table 1). The recommended number of key points is at least 1000 per image, and the minimum value observed in this study is 9278 . The minimum number of matched key points to calibrate an image is 25 , however the recommended number of matches is at least 1000 per image [29]. As seen in Fig. 2(b), despite the use of high image overlap percentages, there are some areas with weak matches because of the changes in the scene during the image acquisition due to different time of capture and moving objects. Nevertheless, 2212 images out of 2216 are calibrated accurately without acquiring images again in these areas to increase image overlapping.
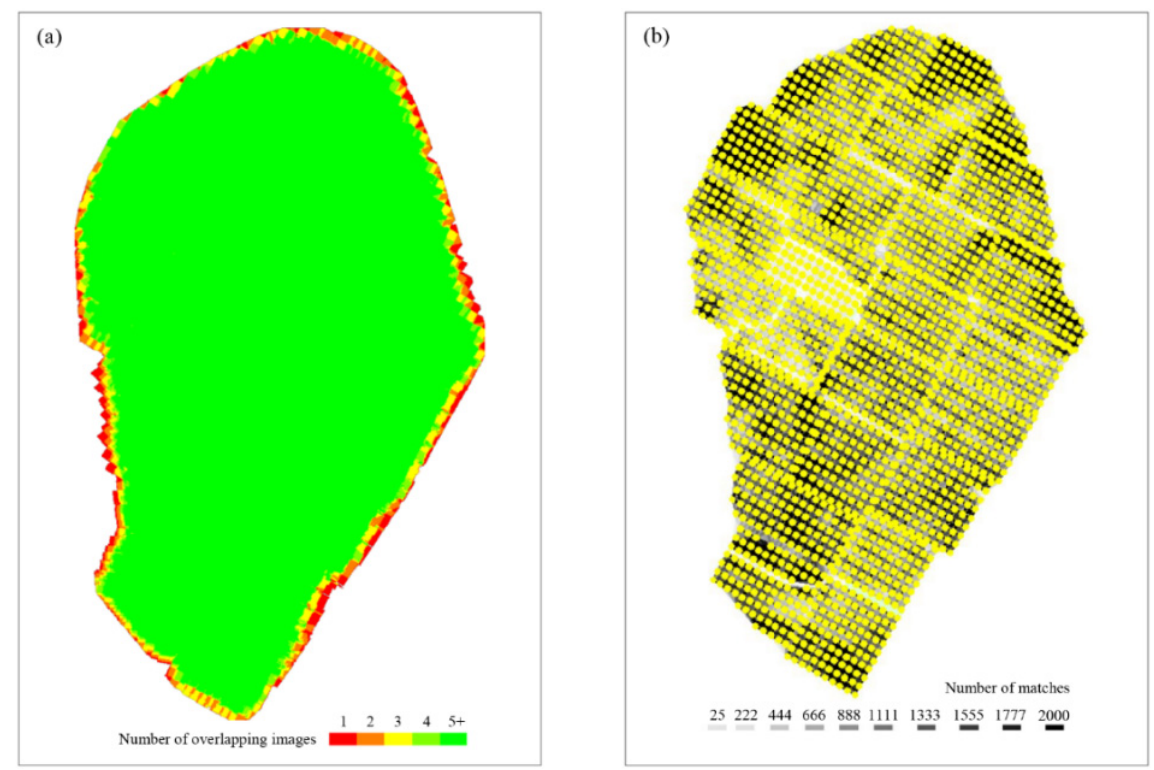

Figure 2: (a) Number of overlapping aerial images calculated for each pixel of the orthomosaic; (b) Determined image positions with links between matched aerial images. 
Table 1: Number of key and matched key points per image.

\begin{tabular}{lll}
\hline & $\begin{array}{l}\text { Number of key } \\
\text { points per image }\end{array}$ & $\begin{array}{l}\text { Number of matched } \\
\text { key points per image }\end{array}$ \\
\hline Median & 25986 & 5585 \\
Mean & 9278 & 154 \\
Max & 51162 & 15828 \\
Mean & 26008 & 5816 \\
\hline
\end{tabular}

The results of the topographic survey for this flood risk area using UAV imagery is shown in Fig. 3. The densified point cloud consists of approximately 600 million 3D points, and the average densities are about 116 points $/ \mathrm{m}^{2}$ and 0.22 points/pixel. The ground sampling distance of the generated DSM is $4.32 \mathrm{~cm} / \mathrm{pixel}$. This resolution is sufficient to be used in 2D hydrodynamic model analyses. Moreover, the unified high-resolution orthophoto of the mapping area can be used in the preparation of flood hazard and flood risk maps to clearly identify the effects of possible flood events. However, the areas with low image overlaps at the boundaries of DSM should not be included in the computational 2D flow area of hydrodynamic models.

The absolute accuracy of the generated DSM is evaluated with 3 check points measured by with a Leica Viva GS15 GNSS receiver using CORS-TR network. Differences between the location data of the check points and the positions of these points in the DSM are summarized in Table 2. The root-mean-square error (RMSE) values for the 3 check points in $\mathrm{x}-, \mathrm{y}-$, and z- coordinates are calculated as about $3.5 \mathrm{~cm}, 6.3 \mathrm{~cm}$., and $12.7 \mathrm{~cm}$., respectively. This corresponds to a RMSE of $5.1 \mathrm{~cm}$ for the horizontal plane and a RMSE of $8.5 \mathrm{~cm}$ for all coordinates of the generated DSM. The results indicate the efficiency of using GCPs to decrease locational uncertainty in image georeferencing. These error values are similar to those obtained in other studies that conducted similar surveys using UAV photogrammetry, and they are considered as acceptable for mapping the results of hydrodynamic model simulations related to flood extents, water depths, and flow velocities [18], [28], [30].

In this study, topographic data processing is performed on a Windows 10 (64 bit) desktop equipped with an Inter Core i7-6700 processor operating at $3.40 \mathrm{GHz}, 32 \mathrm{~GB}$ memory, and a NVIDIA GeForce GTX 680 graphics card. The time spent for initial processing, point cloud densification, 3D textured mesh generation, DSM generation, and orthophoto generation are $173 \mathrm{~min}, 1094 \mathrm{~min}, 79 \mathrm{~min}, 387 \mathrm{~min}$, and $132 \mathrm{~min}$, respectively. Additionally, it takes approximately 5 hours to upload the position data of the GCPs, identify their exact locations on multiple images, and reoptimize the process.

Table 2: Localisation accuracy per check point and RMS errors in the three coordinate directions.

\begin{tabular}{lccc}
\hline Check point ID & Error $\mathrm{X}(\mathrm{m})$ & Error $\mathrm{Y}(\mathrm{m})$ & Error $\mathrm{Z}(\mathrm{m})$ \\
\hline 1 & -0.027 & -0.024 & 0.168 \\
2 & -0.041 & -0.107 & -0.030 \\
3 & 0.036 & -0.002 & -0.140 \\
RMSE & 0.035 & 0.063 & 0.127 \\
\hline
\end{tabular}



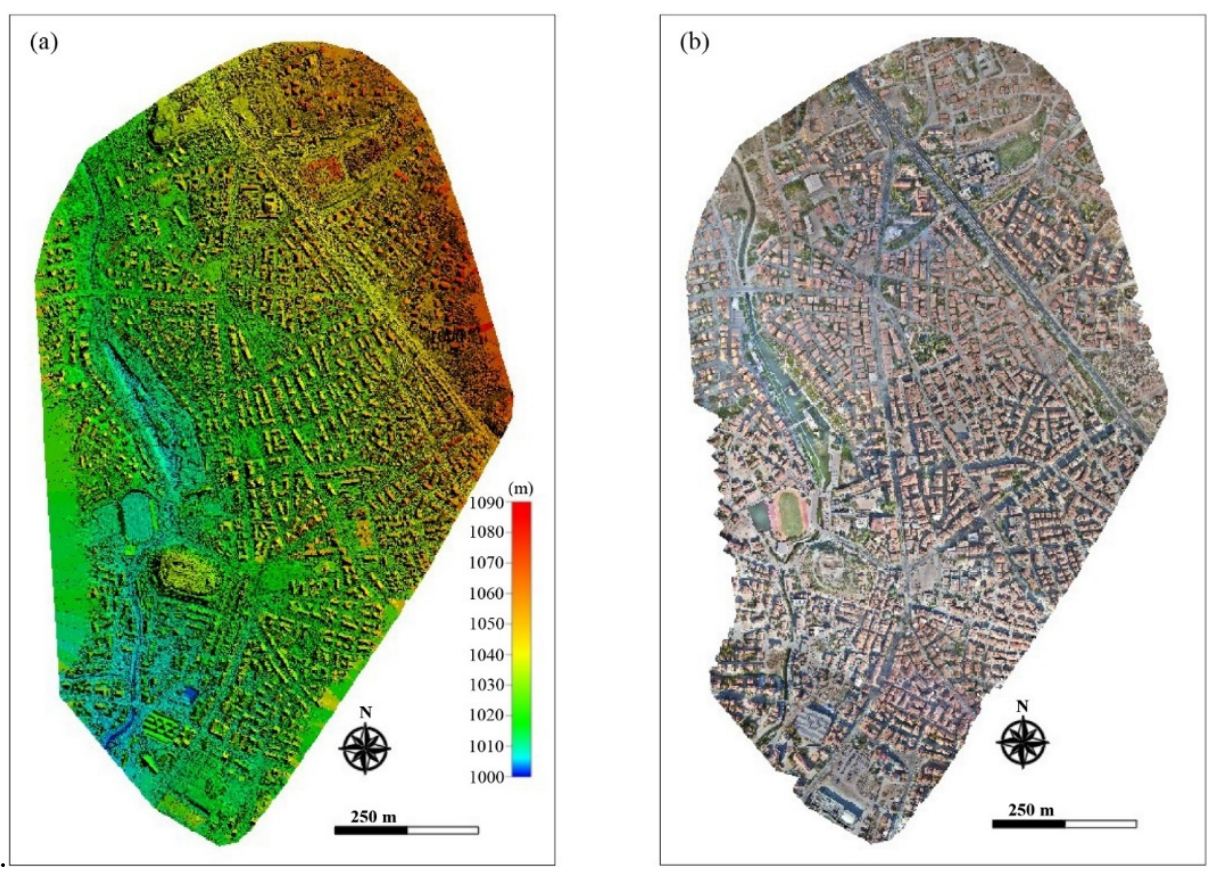

Figure 3: Results of the topographic survey for the flood risk area. (a) DSM; (b) Orthophoto.

The GCP measurements and flight operations on the field are completed in approximately 4 days. Thus, the targeted high-resolution DSM covering an area of $3.42 \mathrm{~km}^{2}$ is generated within one week in contrast to the much more time-consuming ground-based surveying techniques to map such a complex urban area with intensive vehicle and pedestrian traffic. Remote sensing based on UAV imagery could be evaluated as a noteworthy alterative to insitu observations and conventional remote sensing driven by satellite and airborne-based remote sensing platforms in terms of cost-effective acquisition of high-resolution spatial data [31].

Before using the generated DSM in 2D hydrodynamic model analyses, the terrain should be modified for the existing flow obstructing structures, such as the Hastane overpass on the Ankara-Kayseri road, the road culverts on Kilicozu Creek, the pedestrian overpasses on the Ankara-Kayseri road and on Kilicozu Creek. If these structures are not removed when doing flood modelling, they will appear as barriers through which water cannot pass. In this purpose, the dimensions and locations of existing hydraulic structures should be determined by terrestrial measurements, and these structures should be removed from the generated surface model in GIS environment. Then, these hydraulically significant elements should be accurately remodelled on the modified terrain for a designated 2D flow area using the area connection tools of hydraulic models. Thus, the third dimension required to ensure water flow through these structures will be provided for modelling the inundation process in a $2 \mathrm{D}$ flow area.

\section{CONCLUSIONS}

This study presents the steps carried out to generate a high-resolution DSM with a high order of accuracy for a complex urban area using UAV-based aerial photography. From 25 times 
autopilot missions, 2219 aerial images are acquired across an urban area of $3.42 \mathrm{~km}^{2}$ using a DJI Matrice 600 UAV-drone, and a data processing procedure is executed with the preinstalled GCPs in Pix4DMapper Pro software. After elapsing about one week for GCP establishments, flight operations, and data processing, a georeferenced orthophoto and a DSM with a ground sampling distance of $4.21 \mathrm{~cm} /$ pixel are produced. The localization accuracy (RMSE) of the produced model is determined as $5.1 \mathrm{~cm}$ and $12.7 \mathrm{~cm}$ for the horizontal and vertical planes, respectively, by comparison with the coordinates of preinstalled check points.

The results demonstrate that high-resolution digital surface models, which can be used as inputs in 2D flood models for preparing flood hazard and flood risk maps, can be produced with relatively low cost and within a short time using UAV imagery. However, before using the produced DSM in a 2D hydrodynamic model, false blockages to flow in the DSM, such as bridges and culverts, should be removed, and these hydraulically significant elements should be accurately reconstructed in the $2 \mathrm{D}$ flow area of hydrodynamics models to ensure water flow through these structures.

The major deficiencies of UAV technology not being used in larger areas are short flight times due to limited battery capacity and remote-control range of devices. If UAV technology improves on these issues, it will also be possible to accurately monitor flood events in realtime for larger scale areas. The hydraulics of monitored floods are indispensable for the calibration process of $2 \mathrm{D}$ hydrodynamic models to be used in the preparation of flood risk management plans. It should be noted that rapidly evolving UAV technology will increase its potential of use in hydraulic modelling by enhancing these issues.

\section{ACKNOWLEDGEMENTS}

This work was supported by the Ahi Evran University Scientific Research Projects Coordination Unit [grant number MMF.A3.17.004]. The UAV flight operations were conducted under the permission of the Governorship of Kirsehir [permission number 49249874.53197.(91244).2017].

\section{REFERENCES}

[1] Sole, A., Giosa, L., Albano, R. \& Cantisani, A., The laser scan data as a key element in the hydraulic flood modelling in urban areas. Proceedings of the 29th Urban Data Management Symposium, pp. 65-70, 2013.

[2] Schumann, G., Matgen, P., Cutler, M.E.J., Black, A., Hoffmann, L. \& Pfister, L., Comparison of remotely sensed water stages from LiDAR, topographic contours and SRTM. ISPRS Journal of Photogrammetry and Remote Sensing, 63(3), pp. 283-296, 2008. https://doi.org/10.1016/j.isprsjprs.2007.09.004.

[3] Sanders, B.F., Evaluation of on-line DEMs for flood inundation modeling. Advances in Water Resources, 30(8), pp. 1831-1843, 2007. https://doi.org/10.1016/j.advwatres. 2007.02.005.

[4] Casas, A., Benito, G., Thorndycraft, V.R. \& Rico, M., The topographic data source of digital terrain models as a key element in the accuracy of hydraulic flood modelling. Earth Surface Processes and Landforms, 31(4), pp. 444-456, 2006. https://doi.org/10.1002/esp.1278.

[5] Wilson, M.D. \& Atkinson, P.M., A comparison of remotely sensed elevation data sets for flood inundation modeling. Proceedings of the 7th International Conference on Geocomputation, 2013. 
[6] Cook, A. \& Merwade, V., Effect of topographic data, geometric configuration and modeling approach on flood inundation mapping. Journal of Hydrology, 377(1), pp. 131-142, 2009. https://doi.org/10.1016/j.jhydrol.2009.08.015.

[7] Smith, M.J., Edwards, E.P., Priestnall, G. \& Bates, P.D., Exploitation of New Data Types to Create Digital Surface Models for Flood Inundation Modeling, FRMRC Research Report UR3, 2006.

[8] Els, Z., Data availability and requirements for flood hazard mapping. PositionIT. January/February 2013.

[9] d'Oleire-Oltmanns, S., Marzolff, I., Peter, K.D. \& Ries, J.B., Unmanned aerial vehicle (UAV) for monitoring soil erosion in Morocco. Remote Sensing, 4(11), pp. 33903416, 2012. https://doi.org/10.3390/rs4113390.

[10] Niethammer, U., James, M.R., Rothmund, S., Travelletti, J. \& Joswig, M., UAV-based remote sensing of the Super-Sauze landslide: Evaluation and results. Engineering Geology, 128, pp. 2-11, 2012. https://doi.org/10.1016/j.enggeo.2011.03.012.

[11] Turner, D., Lucieer, A. \& Watson, C., An automated technique for generating georectified mosaics from ultra-high resolution unmanned aerial vehicle (UAV) imagery, based on structure from motion (SfM) point clouds. Remote Sensing, 4(5), pp. 1392-1410, 2012. https://doi.org/10.3390/rs4051392.

[12] Turner, D., Lucieer, A. \& De Jong, S.M., Time series analysis of landslide dynamics using an unmanned aerial vehicle (UAV). Remote Sensing, 7(2), pp. 1736-1757, 2015. https://doi.org/10.3390/rs70201736.

[13] Su, T.C. \& Chou, H.T., Application of multispectral sensors carried on unmanned aerial vehicle (UAV) to trophic state mapping of small reservoirs: A case study of Tain-Pu reservoir in Kinmen, Taiwan. Remote Sensing, 7(8), pp. 10078-10097, 2015. https://doi.org/10.3390/rs70810078.

[14] Xu, Z. et al., Development of an UAS for post-earthquake disaster surveying and its application in Ms7. 0 Lushan Earthquake, Sichuan, China. Computers \& Geosciences, 68, pp. 22-30, 2014. https://doi.org/10.1016/j.cageo.2014.04.001.

[15] Leica Viva GS 15. www.leica-geosystems.com/products/gnss-systems/smartantennas/leica-viva-gs15. Accessed on: 15 Jan. 2018.

[16] CORS-TR. http://cors-tr.iku.edu.tr/ENGLISH.htm. Accessed on: 15 Jan. 2018.

[17] DJI Matrice 600. www.dji.com/matrice600. Accessed on: 15 Jan. 2018.

[18] Suh, J. \& Choi, Y., Mapping hazardous mining-induced sinkhole subsidence using unmanned aerial vehicle (drone) photogrammetry. Environmental Earth Sciences, 76(4), p. 144, 2017. https://doi.org/10.1007/s12665-017-6458-3.

[19] Siebert, S. \& Teizer, J., Mobile 3D mapping for surveying earthwork projects using an Unmanned Aerial Vehicle (UAV) system. Automation in Construction, 41, pp. 1-14, 2014. https://doi.org/10.1016/j.autcon.2014.01.004.

[20] UgCS. www.ugcs.com/en/ugcs_features_applications. Accessed on: 15 Jan. 2018.

[21] UgCS for DJI app. www.ugcs.com/en/page/ugcs-for-dji. Accessed on: 15 Jan. 2018.

[22] How the onboard GPS affects the accuracy of a project? Pix4D Support. https://support.pix4d.com/hc/en-us/articles/202558909\#gsc.tab=0. Accessed on: 15 Jan. 2018.

[23] Mendes, T., Henriques, S., Catalao, J., Redweik, P. \& Vieira, G., Photogrammetry with UAV's: quality assessment of open-source software for generation of ortophotos and digital surface models. Proceedings of VIII Conferencia Nacional De Cartografia e Geodesia, pp. 29-30, 2015. 
[24] Küng, O. et al., The accuracy of automatic photogrammetric techniques on ultra-light UAV imagery. Proceedings of the International Conference on Unmanned Aerial Vehicle in Geomatics (UAV-g), 2011.

[25] Pix4DMapper Pro. www.pix4d.com/product/pix4dmapper-photogrammetrysoftware/. Accessed on: 15 Jan. 2018.

[26] Webb, J.A. \& Aggarwal, J.K., Structure from motion of rigid and jointed objects. Artificial Intelligence, 19(1), pp. 107-130, 1982. https://doi.org/10.1016/00043702(82)90023-6.

[27] Schenk, T., Towards automatic aerial triangulation. ISPRS Journal of Photogrammetry and Remote Sensing, 52(3), pp. 110-121, 1997. https://doi.org/10.1016/S09242716(97)00007-5.

[28] Manatunga, U.I., Munasinghe, N. \& Premasiri, H.M.R., Development of a methodology to map railway lines and surrounding land use using UAVs. Proceedings of the International Symposium on Earth Resources Management and Environment, 2017.

[29] 2D key points table - 2D key point matches graph; Pix4D Support. https://support.pix4d.com/hc/en-us/articles/202558689\#label7. Accessed on: 15 Jan. 2018.

[30] Lee, S. \& Choi, Y., Topographic survey at small-scale open-pit mines using a popular rotary-wing unmanned aerial vehicle (drone). Tunnel and Underground Space, 25(5), pp. 462-469, 2015. https://doi.org/10.7474/TUS.2015.25.5.462.

[31] Shuchman, R.A., Sayers, M.J. \& Brooks, C.N., Mapping and monitoring the extent of submerged aquatic vegetation in the Laurentian Great Lakes with multi-scale satellite remote sensing. Journal of Great Lakes Research,39(1), pp. 78-89, 2013. https://doi.org/10.1016/j.jglr.2013.05.006. 